\title{
The Inflationary Universe
}

\section{John Ellis, CERN, Geneva}

\begin{abstract}
Why is the Universe so large and old, while having a density today close to the critical density required to close it, and appearing homogeneous and isotropic on very large scales? These puzzles would be solved if the Universe had undergone an early period of exponential inflationary expansion, perhaps driven by an effective cosmological constant appearing in a field theory of particles.
\end{abstract}

The Universe we see around us today is very large and very old, appears homogeneous and isotropic on large scales, and is expanding perhaps eternally. These are the basic facts of cosmological life. Until recently one had to accept them as given, but in the past few years it has become possible to explain them as consequences of a theory applying particle physics ideas to the evolution of the very early Universe. This new inflationary cosmology not only explains the large-scale uniformity of the Universe today, but also provides a possible origin for the small perturbations in the early Universe which have subsequently evolved into galaxies and galactic clusters. It also predicts that over $90 \%$ of the matter in the Universe remains to be observed: this "dark matter" may play a key role in the formation of galaxies and in their stability today.

In this review I first formulate the puzzles which inflationary cosmology resolves, then outline the basic particle physics mechanism for inflation and describe some of the observational consequences. Finally I mention some of the unresolved problems in inflationary cosmology.

The present day expansion of the Universe was discovered by Hubble in the 1920's when he observed the redshifts of distant galaxies. This observational discovery meshed very well with Einstein's realization a few years earlier that the equations of his General Theory of Relativity did not have a static solution which was stable. Einstein had tried to evade this conclusion by introducing a new term into his equations called the cosmological constant - something he subsequently regarded as his greatest mistake. Hubble's redshift observations suggested that one should instead focus on the expanding models of the Universe known to be solutions of Einstein's equations without a cosmological constant. Subsequently two other major pieces of evidence have confirmed the expanding Universe as the standard cosmological model. Like any other gas, one would have expected the Universe to have been hotter when it was more compressed during earlier stages of its expansion. On the basis of this simple remark, Gamow suggested that the Universe today should contain microwave radiation left over from the time when the size of the Universe was less than a thousandth of its present value, and the ionized plasma condensed into neutral atoms as it expanded and cooled.

Just such a cosmic microwave background radiation was observed by Penzias and Wilson over 20 years ago ${ }^{1}$ ) and is generally taken as confirmation that the Universe was once more than a thousand times smaller that it is today. Another astrophysical observation, namely that about a quarter of the observed matter in the Universe is helium-4 ${ }^{2}$ ) suggests that it was once a million times smaller still. It is difficult to explain this and the abundances of other light elements except as the result of nuclear fusion in the very early Universe when its temperature was $10^{9}$ to $10^{10} \mathrm{~K}$ - primordial nucleosynthesis. Thus it seems that the present expansion of the Universe can be extrapolated backwards to an epoch when the size of the Universe was less than $10^{-9}$ of its present value. If one seeks to go further back, there are theorems based on General Relativity indicating that in the past history of every part of the Universe there must have been a singular point at which the known laws of physics broke down the Big Bang.

There are some baffling features of this standard Big Bang theory of the Universe which we should like to understand. The only fundamental parameter appearing in Einstein's equations which govern the expansion of the Universe is Newton's constant $G_{N}$, familiar from the classical gravitational attraction between two massive objects: $G_{N} m_{1} m_{2}$ / $r^{2}$. Newton's constant has the dimension of (mass) $)^{-2}$, and the mass value is often called the Planck mass $M_{\mathrm{p}}$. It corresponds by special relativity to an energy of about $1.2 \times 10^{19} \mathrm{GeV}$.

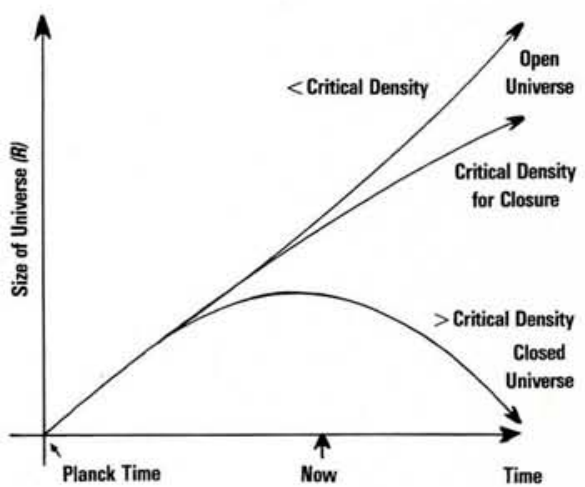

Fig. 1 - Illustration of the expansion of Universes with densities below, equal to and above the critical closure density. It is surprising that our Universe is so close to the closure density, despite being much older than the Planck time.

The Planck mass is related by the uncertainty principle to a Compton wavelength called the Planck length $\ell_{\mathrm{p}}$ of the order of $10^{-33} \mathrm{~cm}$, which would be traversed by light in the Planck time $t_{\mathrm{p}}$ of the order of $10^{-43}$ seconds. Since Einstein's equations only contain Newton's constant and hence these Planck scales, how is it that the solution to those equations which is our Universe today is so much larger than the Planck length, and is about $10^{60}$ times older than the Planck time?

A related puzzle concerns the present density of the Universe which is within about an order of magnitude of the critical density required to close it, despite its enormous expansion since the Big Bang. For the density to be so close to critical today it must have been finetuned to within about $10^{-60}$ of the critical density back when the Universe's age was of the order of the Planck time. If not so finely tuned, the Universe would either have been overdense, halted its expansion some time ago and started contracting under its own weight, or it would be underdense and be expanding much faster than it is today, as seen in Fig. 1. Another puzzle is why has the Universe grown isotropically and homogeneously over distances much larger than the particle horizon, the longest distance which a light wave could have covered since the start of the conventional Big Bang Universe? This dilemma is posed most acutely by the microwave background radiation, which is very isotropic today, implying that its intensity at the epoch of its production must have varied by less than one part in $10^{4}$ over distances a hundred times longer than the horizon scale at that epoch. There are other cosmological puzzles, such as the unexpectedly low density of such unwanted elementary particles as 
magnetic monopoles. I will not discuss them in detail here, since they are almost exclusively a headache for particle physicists, but historically they are important in that they motivated the particle physicists' preoccupation with cosmological problems and this in turn led to the concept of the inflationary Universe.

If we go back to the very early Universe before primordial nucleosynthesis, the temperature and typical kinetic energies were so high that any description of matter should be in terms of the elementary particles and their degrees of freedom. Grand unified theories of elementary particle interactions have already demonstrated their relevance by providing a qualitative mechanism for creating a small matter - antimatter asymmetry in the very early Universe, consistent with the small fraction of baryons relative to photons in the Universe today, and the absence of large amounts of antimatter.

In Einstein's equations, the expansion of the Universe is driven by its energy density for which elementary particles offer three alternative dominant forms: relativistic particles, non-relativistic particles, or a constant vacuum energy analogous to the cosmological constant introduced by Einstein. Nowadays, matter in the Universe is predominantly nonrelativistic although relativistic particles are believed to have dominated back when it was an ionized plasma. In both the relativistic and non-relativistic cases, the energy density falls as the temperature falls and the Universe expands. This means that the rate of expansion steadily slows down, even though it may still expand to an infinite extent if the energy density is less than or equal to the critical density required to close the Universe. The situation would have been very different if at some early epoch the energy density was dominated by a constant vacuum energy. In this case the rate of expansion $(1 / R)(d R / d t)$ would have been constant, and hence the size $R$ of the Universe would have expanded exponentially.

Such an exponential expansion is the core idea of inflationary cosmology. Provided the inflationary epoch lasted long enough, the Universe would have expanded far beyond its "natural" Planck size and even if the Universe is at some date to halt and reverse its post-inflationary expansion, only a slight excess in the duration of the exponential expansion would allow it to expand far beyond the size we see today. A Universe in this state would be essentially indistinguishable from one with the critical energy density required to expand out to infini- ty. Thus not only are the great size and age of the Universe reasonable, but it is also "natural" that the density of the Universe should still be close to critical, as observed. We can also understand the great homogeneity of the Universe on scales much larger than the apparent early particle horizon. Before the hypothesized inflationary epoch, the entire observable Universe would have been infinitesimally smaller than in the naive Big Bang picture, as seen in Fig. 2, so that a light wave could easily have crossed it from one side to the other and causality would permit coordination between opposite sides. The Universe would indeed be essentially homogeneous and isotropic during the exponential inflation driven by a constant vacuum energy, and it should be no surprise that it has remained so today. Finally, note that if there were any obnoxious elementary particles such as magnetic monopoles present in the Universe before inflation or during its early stages, the subsequent exponential expansion would have made their separations grow so large that there would remain at most one in the entire observable Universe.
What might then have caused the expansion of the Universe to be controlled for a while by a constant vacuum energy, analogous to the cosmological constant mentioned earlier? The most natural source to consider is a scalar field locked in a coherent configuration different from its minimum energy state. Scalar fields are a general feature of modern gauge theories of the fundamental interactions. They are used to provide the spontaneous symmetry breaking needed to accommodate non-zero masses for the $\mathrm{W}$ and $\mathrm{Z}^{\circ}$ gauge bosons as well as for quarks and leptons. One expects to find associated with them physical scalar particles called Higgs bosons, although none has yet been discovered. Today when the Universe is cold, Higgs fields sit in their lowest energy configurations, breaking the symmetry of the original field equations as illustrated in Fig. 3 where the lowest energy configuration is away from the symmetric zero point. Earlier in the history of the Universe when it was hot, thermal fluctuations would have pushed the Higgs Field up, out of its lowest energy configuration to the symmetric zero point. This is

\section{The University of Troms $\emptyset$ invites applications for a tenured faculty position in atmospheric and space physics at the full professor level}

The Professor will be appointed to teach physics at the undergraduate and graduate level and to conduct original research in solarterrestrial physics. Applications are particularly welcome from candidates with a strong interest in theoretical and/or experimental research connected with the European Incoherent Scatter Facility (EISCAT), the Partial Reflection Experiment (PRE) and the lonospheric Modification Experiment (HEATING).

Salary is NOK 233.820 p.a. gross, of which NOK 4.010 is paid in pension contribution. The Professor is to accept without compensation any changes in scientific duties, pension or retiring age made by law or by the King in agreement with Parliament.

Applicants should submit 5 copies of their list of publications and scientific works (published or unpublished), divided into five groups, each in numbered order, to the University of Troms $\varnothing$ no later than 3 months after the application deadline. If warning is given no later than one month after the application deadline, manuscripts in preparation may also be submitted, but no later than 3 months after the application deadline. Applicants are othervise referred to the current rules for the procedure to be followed in the appointment of full professors.

Further information about the vacant position can be obtained from

Professors E. Leer and J. Trulsen,

Department of Physics, University of Troms $\varnothing$.

P. O. Box 953, N-9001 Troms $\varnothing$, Norway, Tel. : (83) 8606.

In accordance with university policy, applications from women are especially encouraged.

Applications including a complete curriculum vitae, relevant transcripts, and a copy of the publication list, should be addressed to the King and submitted before 20 November 1985 to:

The University of Troms $\varnothing$

P. O. Box 635, N - 9001 Troms $\varnothing$, Norway. 


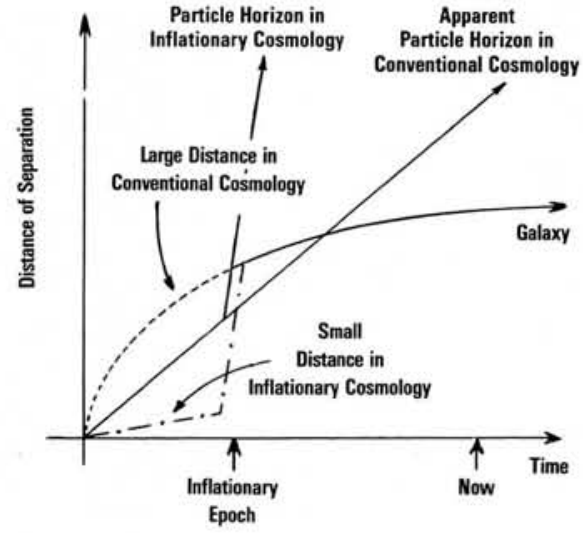

Fig. 2-Different parts of the Universe can be homogeneous if their distance of separation was once smaller than the particle horizon, shown by the full straight lines. In conventional cosmology a distant galaxy would have been outside the apparent particle horizon during most of its history (dotted line). In inflationary cosmology the location of the galaxy would have been much closer at early times, well within the particle horizon. In this case, causality enables us to understand the large-scale homogeneity and isotropy of the Universe.

analogous to the melting of an ordered crystal to become a disordered liquid. Under the right conditions, the Higgs "liquid" could have become supercooled as the Universe expanded, remaining in the disordered symmetric phase although the ordered "solid" phase was energetically favoured.

Precisely because the Higgs field was stuck in an energetically disfavoured "liquid" phase, its configuration would have had a positive vacuum energy $\left(V_{\text {。 }}\right.$ in Fig. 3), and this positive vacuum energy could have driven inflation. At some point, the Higgs "liquid" would finally have completed its transition to the lower energy "solid" phase, and all the latent vacuum energy would have been released to create conventional particles. In order to preserve the previous success of particle physics in explaining primordial matter creation, this should have taken place after the inflationary epoch, so that the precious matter was not subsequently diluted by entropy created when the Higgs vacuum energy converted into particles.

This is the basic inflationary scenario but to make it work in practice is not easy. One must keep the Higgs field supercooled long enough for the Universe to expand to something like its present size, and one must provide a mechanism for the "liquid-to-solid" phase transition to take place eventually. Moreover, this transition must go to completion, since we do not live in a "Swiss Cheese" Universe with liquid holes in it! Early inflationary cosmologists soon found that a first-order Higgs phase transition would never become complete, so a second order transition has since been favoured. In this case inflation takes place after the phase transition has already started, but while the Higgs field is still small and slowly growing.

During this period the Higgs field can perhaps be viewed almost as a classical ball rolling down a hill, as seen in Fig. 3 . To get a sufficiently long inflationary epoch the slope of the hill must be very small, while for matter creation to be possible at the end of inflation, the hill must be quite high so that the Higgs "ball" reaches the bottom of the "hill" with sufficient energy. To reconcile these two requirements we need a hill with a very wide base - in other words the Higgs field order parameter must be as large as possible, say the Planck scale.

A bonus feature of the inflationary concept is that for the first time one can calculate large scale perturbations in the present-day Universe on the basis of microphysics. A thermal or quantum fluctuation in the very early Universe will have inflated to the scale of a galaxy or a galactic cluster today. All inflationary models give spectra of perturbations which are almost independent of the present large astrophysical scale (as previously requested by cosmologists), although early inflationary models yielded perturbations which were too large, a defect traceable to the large slopes of their hills. Creating a sufficiently flat hill requires a degree of fine-tuning of the parameters associated with the Higgs field which seemed unnatural from the point of view of conventional particle physics. However, this fine-tuning could be performed naturally in a supersymmetric particle theory, without fear of higher order (radiative) corrections steepening up the shallow slope that is required.

There are many "existence" proofs for supersymmetric inflationary models. Most of them are based on supergravity, which is the appropriate framework for incorporating supersymmetry into a theory containing the Planck scale associated with gravity. These models are not sufficiently constrained for the magnitude of the galactic perturbations to be determined. However, models with sufficiently small perturbations inflate for much longer than the minimum time needed to expand the Universe to its present size. This means that the Universe will expand much further in the future, and its present density must be observa-

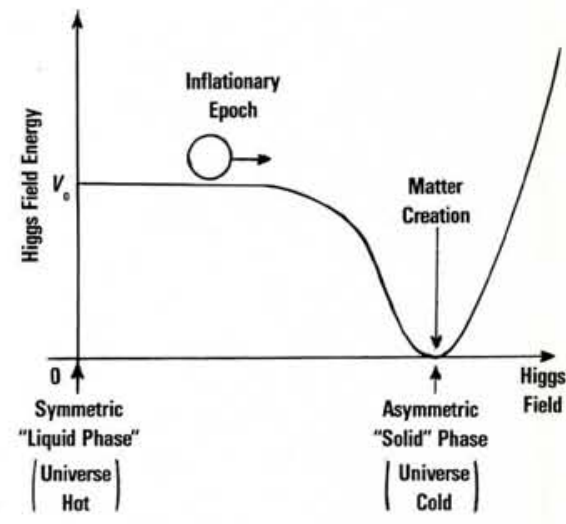

Fig. 3 - Sketch of the Higgs field energy which may have driven inflation. The Higgs field in our Universe may be regarded as a classical "ball" rolling down the "hill" from the symmetric "liquid" phase toward the asymmetric "solid" phase where matter would have been created. While the field energy is close to $V_{o}$ the Universe expands exponentially.

tionally indistinguishable from the critical density in Fig. 1. Since the "known" baryonic matter cannot have a density of much more than a few percent of the critical density, this means there must be a lot of non-baryonic dark matter in the Universe. Supersymmetric theories contain several possible candidates, notably the photino, the neutral spin $1 / 2$ supersymmetric partner of the photon, and the gravitino, the spin $3 / 2$ supersymmetric partner of the graviton. If the dark matter is made of photinos, it may be possible to detect them directly as they pass through sensitive terrestrial detectors, or indirectly as some of them collide and annihilate in the galactic halo. Supersymmetric inflationary cosmology may prove an experimental and observational bonanza.

Problems remain in inflationary cosmology, however. We still cannot derive the required flatness of the hill from fundamental principles; matter creation after inflation is not easy and one must be careful to keep the abundance of unstable supersymmetric particles below observational limits. Nor is it easy to dispose of residual unwanted vacuum energy left over after inflation. Nevertheless, inflation is such an appealing and powerful link between particle physics and cosmology that it is being increasingly accepted by workers in the field.

\section{REFERENCES}

1. Lequeux J., Europhysics News 10 (1979) $1 / 2$.

2. Andrillat H., Europhysics News 12 (1981) $8 / 9$. 